\title{
An in-depth analysis of the RZ Piscium atmosphere
}

\author{
I. S. Potravnov ${ }^{1}$, V. P. Grinin ${ }^{1,2}$, I. V. Ilyin ${ }^{3}$, and D. N. Shakhovskoy ${ }^{4}$ \\ 1 Pulkovo Astronomical Observatory, Russian Academy of Sciences, 196140 Pulkovo, St. Petersburg, Russia \\ e-mail: ilya.astro@gmail.com \\ 2 The Sobolev Astronomical Institute, St. Petersburg University, 198504 Petrodvorets, St. Petersburg, Russia \\ ${ }^{3}$ Leibnitz Institute of Astronomy, 14482 Potsdam, Germany \\ ${ }^{4}$ Crimean Astrophysical Observatory, P. Nauchny, 98409 Crimea, Ukraine
}

Received 28 January 2014 / Accepted 11 February 2014

\begin{abstract}
The star RZ Psc is one of the most unusual members of the UX Ori star family. It demonstrates all properties that are typical of these stars (the light variability, high linear polarization in deep minima, the blueing effect), but until recently there has been no obvious evidence of its youth. Recently, we have shown that RZ Psc is in the transitional evolutionary stage between stars with the primordial and debris disks. In this paper we present the results of the quantitative analysis of the star's spectrum. Using the high-resolution $(R=46000)$ spectrum of RZ Psc obtained with Nordic Optical Telescope, we determined $T_{\text {eff }}=5350 \pm 150 \mathrm{~K}, \log g=4.2 \pm 0.2$, $[\mathrm{M} / \mathrm{H}]=-0.3 \pm 0.05$. These parameters agree well with the same ones as for the stars recently passed the T Tauri star evolutionary stage. This supports our previous suggestion about the evolutionary status of RZ Psc as the post-T Tauri and post-UX Ori star.
\end{abstract}

Key words. stars: individual: RZ Piscium - stars: fundamental parameters - stars: abundances - stars: solar-type

\section{Introduction}

RZ Psc belongs to the UX Ori type star family (UXORs), and it is the coolest member ( $\mathrm{Sp}=\mathrm{K} 0 \mathrm{IV}$, Herbig 1960) of this group. The strong photometric activity of these stars is caused by variable circumstellar (CS) extinction (Grinin et al. 1991) triggered by the small inclination of their CS disks to the line of sight. However, RZ Psc has a number of features that significantly distinguish it from the other UXORs. The Algol-like minima of RZ Psc are very short in time: they last only 1-2 days (Zajtseva 1985; Kardopolov et al. 1980; Pugach 1981; Wenzel 1989). Other UXORs demonstrate such short minima very seldom, since they are typically much longer. Moreover, RZ Psc lies at the Galactic latitude $b \simeq-35^{\circ}$ far from star-formation regions and young stellar associations. Similarly, RZ Psc does not show the classical signatures of youth, such as an emission in $\mathrm{H}_{\alpha}$ line and an infrared (IR) excess in $J H K$ bands. The photometric activity of the RZ Psc was intensively studied (Zajtseva 1985; Kardopolov et al. 1980; Pugach 1981; Wenzel 1989) but up to now only two papers have been devoted to its spectroscopic investigation (Herbig 1960; Kaminskii et al. 2000). In the first paper, the spectral type of RZ Psc was determined, and in the second, the authors determined the atmospheric parameters and chemical composition of the star. The derived parameters $\left(T_{\text {eff }}=5250 \mathrm{~K}, \log g=3.4\right)$ and small depletion of metals (except iron and calcium) led them to conclude that RZ Psc was the moderately evolved population II giant.

In 2009 we started spectroscopic observations of RZ Psc on the Peak Terskol Observatory. The strong Li $6708 \AA$ ( $E W=$ $0.202 \AA$ ) absorption was found in the first RZ Psc spectrum obtained in November 2009. This finding allowed us to roughly estimate the star's age in the range of 10-70 Myr (Grinin et al. 2010). The refined age estimations were based on the analysis of the RZ Psc motion in the gravitational potential of Galaxy. Grinin et al. (2010) and Potravnov \& Grinin (2013) provided the value $t_{k}=25 \pm 5 \mathrm{Myr}$, which significantly exceeds protoplanetary-disk-dissipation timescale (Williams \& Cieza 2011). On the other hand, the results of the RZ Psc spectroscopic monitoring clearly demonstrate the presence of the remnants of the CS gas in the nearest vicinity of the star manifested itself in the NaI resonance doublet lines (Potravnov et al. 2013).

Another recently discovered and intriguing feature of RZ Psc is the comparatively large IR excess at $\lambda>5 \mu \mathrm{m}: 8 \%$ from the star's bolometric luminosity (De Wit et al. 2013). The origin of this excess associated with the presence of warm $(T \approx 500 \mathrm{~K})$ dust produced presumably by the collisional activity in the star's debris disk. The characteristics mentioned above: the age, the presence of the neutral gas in the nearest environment of the star, and high fractional luminosity $L_{\text {ir }} / L_{\star}$ mean that RZ Psc is a very unusual UXOR on the transitional stage between stars with the primordial and debris disks.

The aim of the present work is to revise the stellar atmosphere parameters of RZ Psc by using an echelle spectrum obtained with the Nordic Optical Telescope.

\section{Observations and data reduction}

The high-resolution spectrum of RZ Psc was observed by I.V. Ilyin on August 19, 2013 using the fiber-fed FIES spectrograph at the $2.56 \mathrm{~m}$ Nordic Optical Telescope (NOT) on La Palma, Canarias, Spain. The total exposure time was $3600 \mathrm{~s}$. The spectrum covers a 3700-7300 $\AA$ range, but for the further analysis we use only a 4200-7300 $\AA$ spectral range due to low signal-tonoise ratio $(\mathrm{S} / \mathrm{N})$ in the blue. The fiber diameter is $1.1^{\prime \prime}$ projected on the sky. The resolving power is about 46000 and the $\mathrm{S} / \mathrm{N}$ of 
the extracted spectra is about 75 after a three-point smoothing average.

The echelle spectrum was reduced by using FIEStool pipeline dedicated to the FIES spectrograph with the standard image processing steps applied ${ }^{1}$. The continuum normalization was done with the aid of $2 \mathrm{D}$ smoothing splines applied first in the cross-dispersion direction, then in the wavelength direction. A fit-and-clip algorithm was used to mask out spectral lines from the fit by using robust estimators of the noise, which extracts only the symmetrical part of the continuum noise distribution.

The orders were rectified by preserving the original nonlinear wavelength scale of the pixels. The overlapping parts of the orders were rebinned in accordance with the wavelength of the previous spectral order, and a weighted average between them was obtained. The final step was the spectrum linearization and manual continuum correction. The telluric lines in the spectrum were removed by using the spectrum of the hot rapidly rotating $\operatorname{star} \theta$ And. The simultaneous photometric observations were carried out by D.N. Shakhovskoy using the AZT-11 telescope of the Crimean Astrophysical Observatory.

\section{Quantitative analysis of the spectrum}

The photometric observations showed that RS Psc was in the bright state in the night of the spectral observations: $V=11$. 65 . This implies the absence of a large amount of the CS matter on the line of sight on that night. This fact and the absence of clear accretion traces that can produce veiling of the photospheric lines make our attempt to determine RZ Psc atmospheric parameters quite reliable.

We used the IDL-based tool SME (Valenti \& Piskunov 1996) for the RZ Psc atmospheric parameter determination. This software package allows us to determine stellar parameters by matching the observed spectrum with the grid of calculated synthetic one. The nonlinear least-squares algorithm can solve for the stellar parameters that provide the best agreement between observed and theoretical spectra. In our analysis, we used several spectral regions, including two hydrogen lines, $\mathrm{H}_{\alpha}$ and $\mathrm{H}_{\beta}$, magnesium MgIb triplet, as well as numerous metallic lines. The strong resonance sodium doublet $\mathrm{NaI} D_{1,2}$ was eliminated from our analysis due to its noticeable variability that was recently discovered (Potravnov et al. 2013). The atomic parameters were taken from the VALD atomic line database (Piskunov et al. 1995; Kupka et al. 1999). We used the MARCS atmospheric model grid's (Gustafsson et al. 2008) built-in SME for synthetic spectra calculations.

The broad wings of the $\mathrm{H}_{\alpha}$ line, which are mainly sensitive to $T_{\text {eff }}$, were used for the initial determination of the effective temperature. By varying $T_{\text {eff }}$ we obtained good agreement with the observations in the line wings at $T_{\text {eff }}=5350 \mathrm{~K}$. The deviation of the observed line core from the theoretical prediction indicates the existence of the weak $\mathrm{H}_{\alpha}$ emission (Potravnov et al. 2013). The additional confirmation of our temperature determination comes from the good match of the $\mathrm{H}_{\beta}$ line profile, which was less disturbed by emission. The comparison of the observed $\mathrm{H}_{\alpha}$ and $\mathrm{H}_{\beta}$ profiles with the best-fit model is presented in Figs. 1 and 2. Hereafter, the wavelength is given in the stellar reference frame.

\footnotetext{
1 http://www.not.iac.es/instruments/fies/fiestool/ FIEStool-manual-1.0.pdf
}

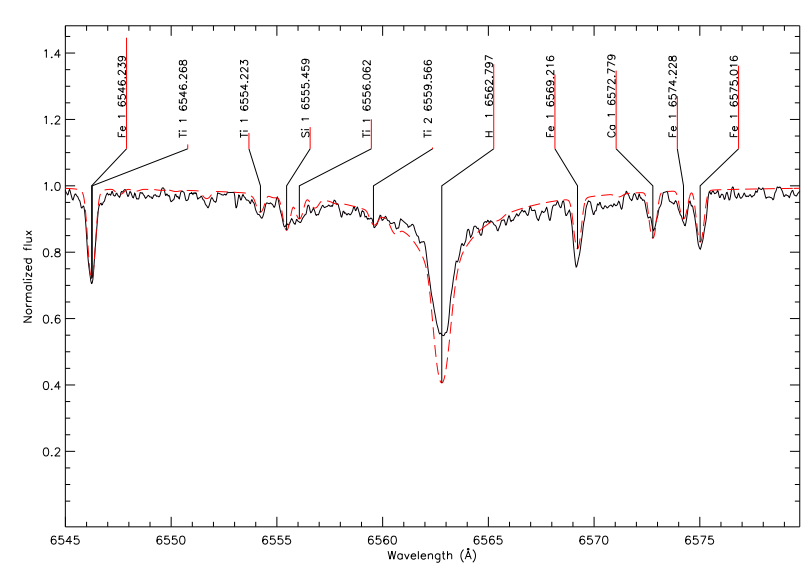

Fig. 1. $\mathrm{H}_{\alpha}$ line in the RZ Psc spectrum (thin line). The synthetic spectrum is shown with the dashed line.

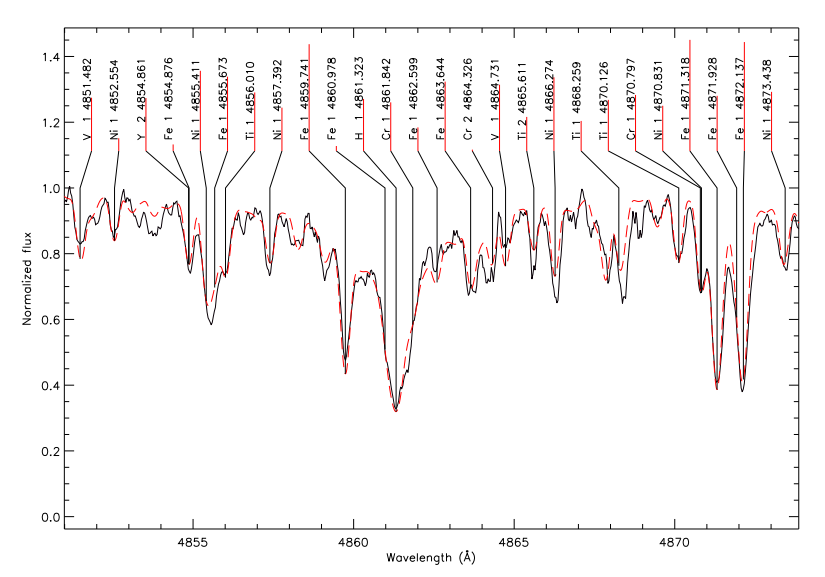

Fig. 2. $\mathrm{H}_{\beta}$ line in the RZ Psc spectrum (thin line). The synthetic spectrum is plotted by the dashed line.

Table 1. Atmospheric parameters derived for RZ Psc.

\begin{tabular}{ll}
\hline \hline Parameter & Value \\
\hline$T_{\text {eff }}$ & $5350 \pm 150 \mathrm{~K}$ \\
$\log g$ & $4.2 \pm 0.2$ \\
{$[M / H]$} & $-0.3 \pm 0.05$ \\
$V_{\text {turb }}$ & $1.0 \mathrm{~km} \mathrm{~s}^{-1}$ \\
$V$ sin $i$ & $12.0 \pm 0.5 \mathrm{~km} \mathrm{~s}^{-1}$ \\
$V_{\text {mac }}$ & $5.0 \mathrm{~km} \mathrm{~s}^{-1}$ \\
\hline
\end{tabular}

The second SME iteration was performed with a fixed temperature. During the subsequent iterative fit of the spectrum, surface gravity $\log g$, metallicity $[\mathrm{M} / \mathrm{H}]$, microturbulent velocity $V_{\text {mic }}$, radial-tangential macroturbulence $V_{\text {mac }}$, and projected rotational velocity $V \sin i$ were determined. The parameters of the best-fit model are presented in the Table 1 .

The abundances of several chemical elements were determined by varying abundance and fitting the observed profiles using IDL package BinMag $3^{2}$. Several lines of each species we discussed were chosen and fitted. The errors were determined as a standard deviation of measurements sample. The results of the

2 http://www.astro.uu.se/ oleg/ 


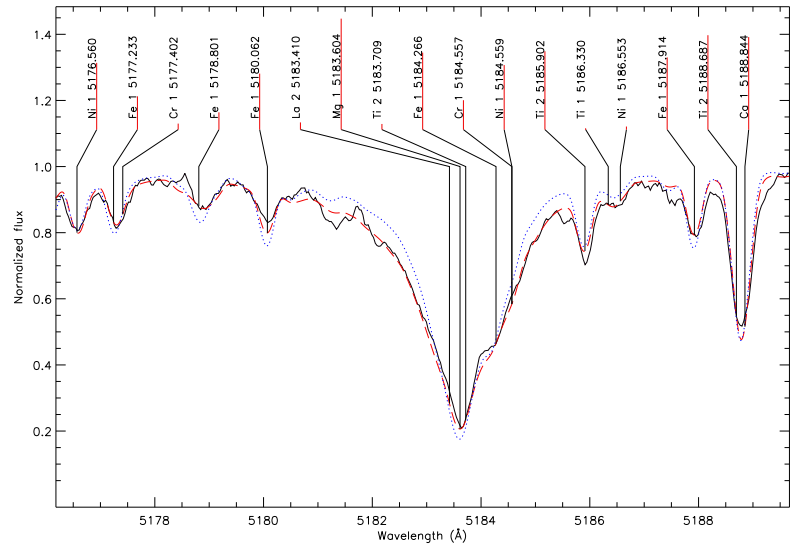

Fig. 3. Spectrum of RZ Psc around the MgIb $5183 \AA$ line (thin line). The synthetic spectrum is showm with the dashed line. The profile calculated with parameters by Kaminskii et al. (2000) and convolved with our value of $V \sin i$ and $V_{\operatorname{mac}}$ shown by the dotted line.

analysis are presented in Fig. 5. We detemined the magnesium abundance using the MgI 5528, 5711, 6318, $6319 \AA$ A lines, which are weaker than MgIb triplet and less sensitive to the surface gravity. The final runs of the surface gravity fitting we made with the fixed magnesium abundance. This allowed us to use the matching between the observed and calculated gravity-sensitive MgIb profiles as the criteria for our $\log g$ choice. The strong lithium line $6708 \AA$ is presented in the RZ Psc spectrum (Grinin et al. 2010) and provides an abundance $[\mathrm{Li} / \mathrm{H}]=1.75 \pm 0.1$ relative to the Sun. Also, we determined the projected rotation velocity $V \sin i=12.0 \pm 0.5 \mathrm{~km} \mathrm{~s}^{-1}$ using SME package. The important byproduct of our analysis is the value of the RZ Psc radial velocity: $V_{r}=-1.2 \pm 0.5 \mathrm{~km} \mathrm{~s}^{-1}$. This value was obtained using the IRAF cross-correlation task fxcor. It is close to our previous estimates $V_{r}=-1.5 \pm 1.0 \mathrm{~km} \mathrm{~s}^{-1}$ (Potravnov \& Grinin 2013).

\section{Discussion and conclusion}

The results of quantitative analysis of the RZ Psc spectrum have confirmed the spectral classification K0 IV given by Herbig. The effective temperature $T_{\text {eff }}$ also agrees with the temperature determined by Kaminskii et al. (2000). However, our value $\log g=$ 4.2 is greater on 0.8 dex than the value of $\log g=3.4$ presented in the paper by Kaminskii et al. (2000). The MgIb $5183 \AA$ A pressurebroadened wings are robust surface gravity tracers in the solartype stars (Fuhrmann et al. 1997). The pressure-broadened wings calculated with our parameters MgIb $5183 \AA$ are in the excellent agreement with the observed wings (Fig. 3). For the comparison, this magnesium line and the part of the spectrum around it were calculated with the parameters by Kaminskii et al. (2000) and plotted in the same figure. One sees that the line wings in their model are narrower than the observed ones. This means that the real value of the surface gravity is higher and close to our value. The synthetic spectrum calculated with our parameters from Table 1 provides a reliable description of the observed metallic lines (Fig. 4). The ionization equilibrium is also confirmed by the relatively good matching of ionized metal lines, some of which are presented in Fig. 4.
In the last few years, several papers were devoted to study of the physical parameters of a carefully selected samples of young stars in the different star-forming regions. In particular, the surface gravities were determined for the weak-line T Tauri stars (WTTS; James et al. 2006; Santos et al. 2008) as well as for the post-T Tauri stars (Viana Almeida et al. 2009). Stars of both types are finishing their contraction phase and approaching the Main Sequence. The surface gravity of RZ Psc agrees well with the results obtained for the members of the young stellar associations.

Another feature of the RZ Psc spectrum is its low metallicity. Kaminskii et al. (2000) reported a "slight metal deficiency". We observed overall metals depletion $[\mathrm{M} / \mathrm{H}]=-0.3$, except individual abundances of calcium and silicon which are close to the solar (Fig. 5). The overabundance of calcium with respect to other metals was noted by Kaminskii et al. (2000) but we cannot confirm the same conclusion for the iron mentioned in their article. It should be noted that while the RZ Psc metallicity differs from the average metallicity obtained for young stellar associations it lies in the range of the observed values (see Fig. 6 in the paper by Viana Almeida et al. 2009). The possible reason of such a chemical composition is an initial composition of the star-formation region where RZ Psc was born. The exoplanet-host star $\iota$ Horologii was ejected from Hyades $\sim 650 \mathrm{Myr}$ ago but still demonstrates the chemical composition close to the original Hyades abundance pattern and thus to the composition of primordial cloud (Vauclair et al. 2008). So, this abundance "birthmark" can be useful at the searches of RZ Psc celestial companions.

Our determination of the projected rotational velocity is almost as twice lower than the value of $23 \mathrm{~km} \mathrm{~s}^{-1}$ presented by Kaminskii et al. (2000). Our value $V \sin i=12 \pm 0.5 \mathrm{~km} \mathrm{~s}^{-1}$ lies near the maximum of rotational velocities histogram for $\mathrm{T}$ Tauri stars (Bouvier et al. 1986) and close to the rotational velocities of G-K dwarfs in Pleiades (Soderblom et al. 1993). One of the possible reasons for the discrepancy in our value of the rotational velocity is the higher spectral resolution of our spectra. Besides, Kaminskii et al. (2000) neglected the line broadening by the macroturbulence. The high value of macroturbulent velocity $\left(V_{\mathrm{mac}}=5 \mathrm{~km} \mathrm{~s}^{-1}\right)$ in the atmosphere of RZ Psc obtained in our paper can be a result of large-scale convective motions. The active convection possibly still not damped in the atmosphere of the comparatively young RZ Psc.

There are also some discrepancies in the radial velocity measurements. As mentioned above, our value of the radial velocity from the NOT spectrum is $V_{r}=-1.2 \pm 0.5 \mathrm{~km} \mathrm{~s}^{-1}$. This value agrees with the earlier measurements by Shevchenko et al. (1993): $V_{r}=-2.0 \pm 2.0 \mathrm{~km} \mathrm{~s}^{-1}$ and differs from the result by Kaminskii et al. (2000): $V_{r}=-11.75 \pm 1.1 \mathrm{~km} \mathrm{~s}^{-1}$. The difference is probably real and can be caused by the Rossiter-McLaughlin effect when the radial velocity variation occurs due to the partial eclipse of the rotating stellar disk by the compact circumstellar clumps (Grinin \& Potravnov 2013). Since our spectroscopic observations were carried out during the star's bright state (out of eclipse), the obtained value $V_{r}=$ $-1.2 \pm 0.5 \mathrm{~km} \mathrm{~s}^{-1}$ is the real radial velocity of the star. It should be noted that the precise value of $V_{r}$ is very important for calculations of the star's kinematic age (Grinin et al. 2010; Potravnov \& Grinin 2013).

The revised parameters of the RZ Psc atmosphere are close to the parameters of WTTS and PTTS. Together with the kinematic age estimation and the lithium abundance this is once more confirmation of the star's youth. The next important step should 


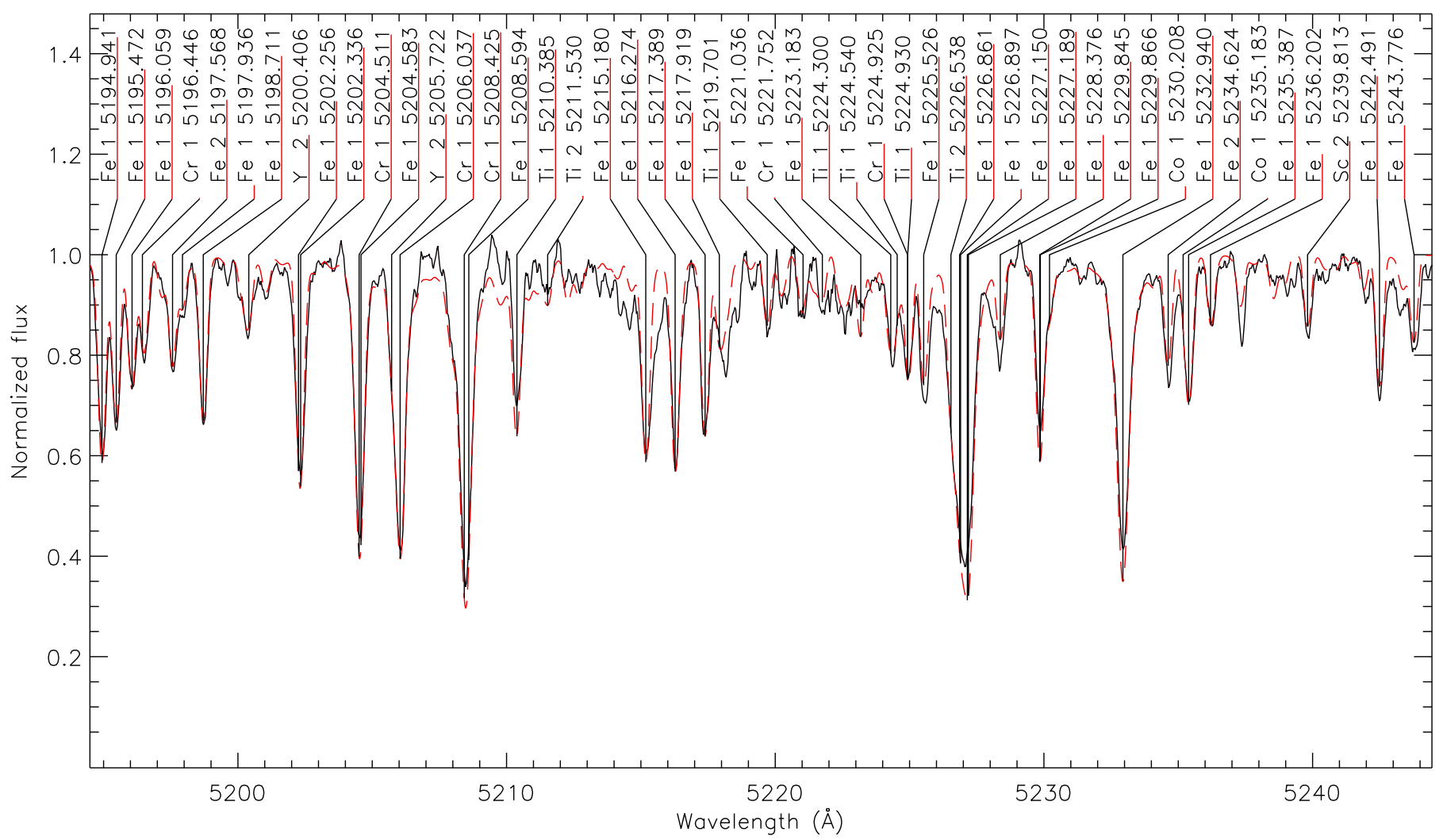

Fig. 4. Part of the RZ Psc spectrum with metals of different ionization states (solid line), together with the synthetic spectrum (dashed line).

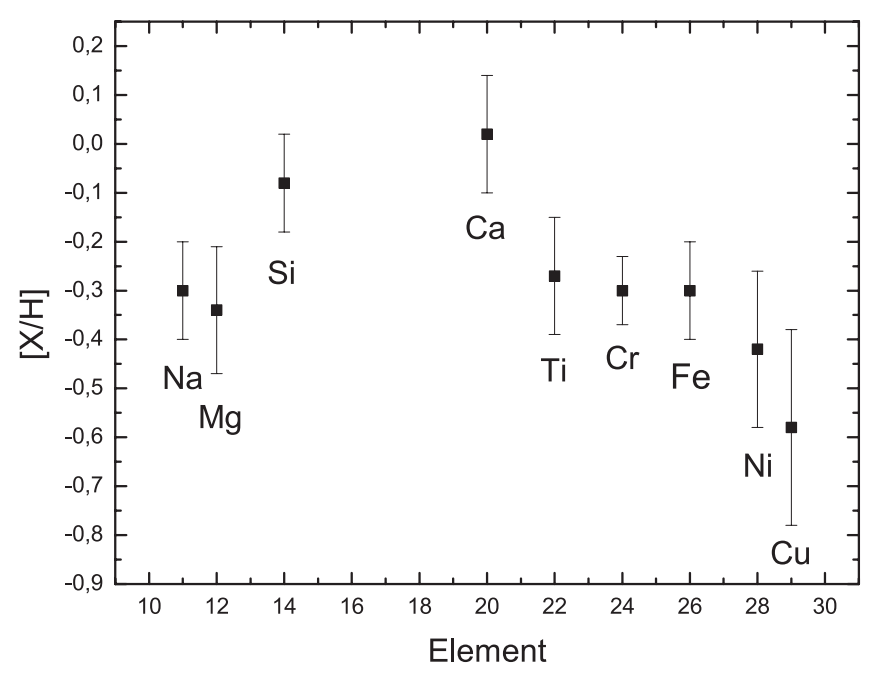

Fig. 5. Individual abundances of several elements in the RZ Psc atmosphere.

be the determination of the RZ Psc mass and luminosity after obtaining the precise distance value from Gaia.

Acknowledgements. We are very grateful to T. A. Ryabchikova and V. V. Tsymbal for their consultation and useful discussion. We are grateful also to the referee M. van den Ancker for his useful comments. This research has been supported by the program P21 "Nonstationary Phenomena in Objects of the Universe" of the Presidium of RAS and grant N.Sh.- 1625.2012.2.

\section{References}

Bouvier, J., Bertout, C., Benz, W., \& Mayor, M. 1986, A\&A, 165, 110 De Wit, W. J., Grinin, V. P., Potravnov, I. S., et al. 2013, A\&A, 553, L1 Fuhrmann, K., Pfeiffer, M., Frank, C., Reetz, J., \& Gehren, T. 1997, A\&A, 323, 909

Grinin, V. P., \& Potravnov, I. S. 2013, Astrophysics, 56, 1

Grinin, V. P., Kiselev, N. N., Chernova, G. P., Minikulov, N. K., \& Voshchinnikov, N. V. 1991, Ap\&SS, 186, 283

Grinin, V. P., Potravnov, I. S., \& Musaev, F. A. 2010, A\&A, 524, A8

Gustafsson, B., Edvardsson, B., Eriksson, K., et al. 2008, A\&A, 486, 951

Herbig, G. H. 1960, ApJ, 131, 632

James, D. J., Melo, C., Santos, N. C., \& Bouvier, J. 2006, A\&A, 446, 971

Kaminskii, B. M., Kovalchuk, G. U., \& Pugach, A. F. 2000, Astron. Rep., 44, 611

Kardopolov, V. I., Sakhanenok, V. V., \& Shutemova, N. A. 1980, Peremennye Zvezdy, 21, 310

Kupka, F., Piskunov, N., Ryabchikova, T. A., Stempels, H. C., \& Weiss, W. W. 1999, A\&AS, 138, 119

Piskunov, N. E., Kupka, F., Ryabchikova, T. A., Weiss, W. W., \& Jeffery, C. S. 1995, A\&AS, 112, 525

Potravnov, I. S., \& Grinin, V. P. 2013, Astron. Lett., 39, 776

Potravnov, I. S., Grinin, V. P., \& Ilyin, I. V. 2013, Astrophysics, 56, 453

Pugach, A. F. 1981, Astrophysics, 17, 47

Santos, N. C., Melo, C., James, D. J., et al. 2008, A\&A, 480, 889

Shevchenko, V. S., Vitrichenko, E. A., Grankin, K. N., Ibragimov, M. A., \& Mel'Nikov, S. Y. 1993, Astron. Lett., 19, 125

Soderblom, D. R., Stauffer, J. R., Hudon, J. D., \& Jones, B. F. 1993, ApJS, 85, 315

Valenti, J. A., \& Piskunov, N. 1996, A\&AS, 118, 595

Vauclair, S., Laymand, M., Bouchy, F., et al. 2008, A\&A, 482, L5

Viana Almeida, P., Santos, N. C., Melo, C., et al. 2009, A\&A, 501, 965

Wenzel, W. 1989, IBVS, 3280, 1

Williams, J. P., \& Cieza, L. A. 2011, ARA\&A, 49, 67

Zajtseva, G. V. 1985, Peremennye Zvezdy, 22, 181 